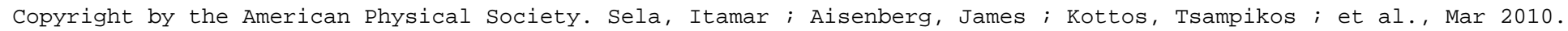

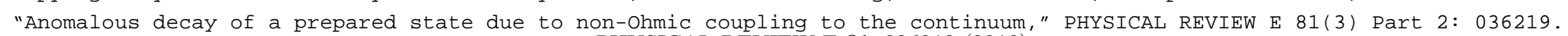
DOI : $10.1103 /$ PhysRevE.81.036219.

\title{
Anomalous decay of a prepared state due to non-Ohmic coupling to the continuum
}

\author{
Itamar Sela, ${ }^{1}$ James Aisenberg, ${ }^{2}$ Tsampikos Kottos, ${ }^{2}$ Alex Elgart, ${ }^{3}$ and Doron Cohen ${ }^{1}$ \\ ${ }^{1}$ Department of Physics, Ben-Gurion University, Beer-Sheva 84105, Israel \\ ${ }^{2}$ Department of Physics, Wesleyan University, Middletown, Connecticut 06459, USA \\ ${ }^{3}$ Department of Mathematics, Virginia Tech, Blacksburg, Virginia 24061, USA
}

(Received 29 May 2009; revised manuscript received 22 December 2009; published 25 March 2010)

\begin{abstract}
We study the decay of a prepared state $E_{0}$ into a continuum $\left\{E_{k}\right\}$ in the case of non-Ohmic models. This means that the coupling is $\left|V_{k, 0}\right| \propto\left|E_{k}-E_{0}\right|^{s-1}$ with $s \neq 1$. We find that irrespective of model details there is a universal generalized Wigner time $t_{0}$ that characterizes the decay of the survival probability $P_{0}(t)$. The generic decay behavior which is implied by rate equation phenomenology is a slowing down stretched exponential, reflecting the gradual resolution of the band profile. But depending on nonuniversal features of the model a power-law decay might take over: it is only for an Ohmic coupling to the continuum that we get a robust exponential decay that is insensitive to the nature of the intracontinuum couplings. The analysis highlights the coexistence of perturbative and nonperturbative features in the dynamics. It turns out that there are special circumstances in which $t_{0}$ is reflected in the spreading process and not only in the survival probability, contrary to the naive linear-response theory expectation.
\end{abstract}

DOI: 10.1103/PhysRevE.81.036219

PACS number(s): 05.45.Mt, 03.65.Sq, 73.23.-b

\section{INTRODUCTION}

The time relaxation of a quantum-mechanical prepared state into a continuum due to some residual interaction is of great interest in many fields of physics. Applications can be found in areas as diverse as nuclear [1], atomic, and molecular physics [2] to quantum information [3], solid-state physics $[4,5]$, and quantum chaos [6]. The most fundamental measure characterizing the time relaxation process is the socalled survival probability $P_{0}(t)$, defined as the probability not to decay before time $t$.

The study of $P_{0}(t)$ goes back to the work of Weisskopf and Wigner $[7,8]$ regarding the decay of a bound state into a continuum. They have found that $P_{0}(t)$ follows an exponential decay $P_{0}(t)=\exp \left(-t / t_{0}\right)$, with rate $1 / t_{0}$ given by the Fermi golden rule (FGR).

Following Wigner, many studies have adopted random matrix theory (RMT) modeling [9-13] for the investigation of $P_{0}(t)$, highlighting the importance of the statistical properties of the spectrum [13], both in the semiclassical [14-16] and in the many-body context [17] where nonexponential decay such as $P_{0}(t) \sim \exp (-\sqrt{t})$ may arise.

Despite the interest in specific problems where deviations from the Wigner theory arise, a theoretical investigation of the time relaxation for prototype RMT models is still missing and also the general (not model specific) perspective is lacking. This unbalanced situation should be contrasted with the arena of spectral statistics [18], where one can find on the one hand elaborated mathematical studies of generics RMT models and on the other hand system specific investigations that explore nonuniversal features that cannot be captured by RMT.

In this paper we would like to explore the limits of universality in decay problems using an RMT perspective. Specifically we explore the decay of an initially prepared state into the continuum assuming non-Ohmic rather than Ohmic circumstances. The latter notions are precisely defined in the next section. We show that the survival probability $P_{0}(t)$ $=g\left(t / t_{0}\right)$ is characterized by a generalized Wigner decay time $t_{0}$ that depends in a nonlinear way on the strength of the coupling. We also establish that the scaling function $g$ has distinct universal and nonuniversal features. It is only for Ohmic coupling to the continuum that we get a robust exponential decay that is insensitive to the nature of the intracontinuum couplings. In addition to $P_{0}(t)$ we investigate other characteristics of the evolving wave packet: the variance $\Delta E_{\text {sprd }}(t)$ and the $50 \%$ probability width $\Delta E_{\text {core }}(t)$ of the energy distribution that describe universal and nonuniversal features of its decaying component.

The structure of the paper is as follows: in Sec. II we define the Friedrichs model (FM) [19] and the generalized Wigner model (WM) and discuss the numerical approach used in the subsequent sections. The quantities under investigation and their physical meaning is discussed in Sec. III. In Sec. IV we present an overview of the relevant time scales that dictate the dynamics of our models. In Sec. V we present analytical and numerical results for the local density of states (LDOS) of the FM and of the WM. The study of the wavepacket dynamics is presented in Secs. VI-VIII: first we analyze the decay of the survival probability and later the evolution of the energy distribution. Our conclusions are given at the last Sec. IX, where we also discuss the crossover from the universal to the nonuniversal behavior.

\section{MODELING}

We analyze two models whose dynamics is generated by a Hamiltonian

$$
\mathcal{H}=\mathcal{H}_{0}+V
$$

with $\mathcal{H}_{0}=\operatorname{diag}\left\{E_{n}\right\}$ and $n \in \mathbb{Z}$. The system is prepared initially in the eigenstate corresponding to $E_{0}$, and the coupling to the other levels is characterized by the spectral function 


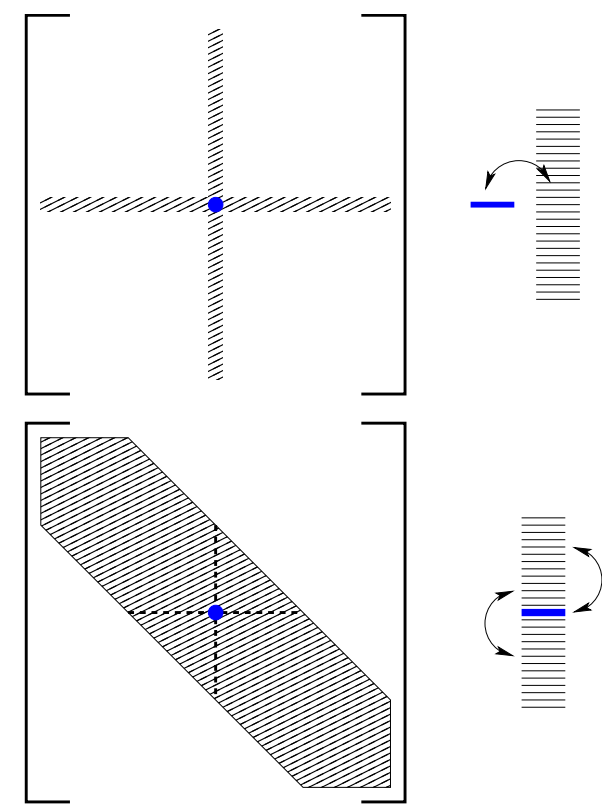

FIG. 1. (Color online) Schematic illustration of the coupling matrix $V_{n m}$ for the FM (upper panel) and the WM (lower panel).

$$
\widetilde{C}(\omega)=\sum_{n \neq 0}\left|V_{n, 0}\right|^{2} 2 \pi \delta\left[\omega-\left(E_{n}-E_{0}\right)\right]
$$

In the so-called Ohmic case $\widetilde{C}(\omega)$ is finite for $\omega \sim 0$ and accordingly FGR suggests a well-defined finite rate of decay. But our interest is in so-called non-Ohmic circumstances for which $\widetilde{C}(\omega)$ either vanishes or diverges as $\omega \rightarrow 0$. To be specific we assume the standard form

$$
\widetilde{C}(\omega)=2 \pi \epsilon^{2}|\omega|^{s-1} e^{-|\omega| / \omega_{c}} .
$$

Note that $\tilde{C}(\omega)$ has $1 /$ time units, and hence $\epsilon$ has $1 /$ time $^{2-s}$ units. In order to define the model one should specify the exponent $s$, the strength of the coupling $\epsilon$, the density of states $\varrho$, and the bandwidth $\omega_{c}=b \varrho^{-1}$. But this is not enough. One also has to specify the couplings between the other levels $E_{n \neq 0}$. Here we distinguish between two cases that are illustrated in Fig. 1 and discussed below.

The FM [19] features a distinguished energy level $E_{0}$ that is coupled to the rest of the levels $E_{n \neq 0}$ by a rank two matrix. This means that the other levels are not coupled. For example the following is a $6 \times 6 \mathrm{FM}$ matrix with $b=2$ :

$$
\mathcal{H}=\left[\begin{array}{cccccc}
E_{0} & V_{01} & V_{02} & 0 & 0 & 0 \\
V_{10} & E_{1} & 0 & 0 & 0 & 0 \\
V_{20} & 0 & E_{2} & 0 & 0 & 0 \\
0 & 0 & 0 & E_{3} & 0 & 0 \\
0 & 0 & 0 & 0 & E_{4} & 0 \\
0 & 0 & 0 & 0 & 0 & E_{5}
\end{array}\right] .
$$

In the FM case the dimensionless bandwidth $b$ defines the effective size of the matrix as $N=b+1$ because out-of-band levels cannot be reached.

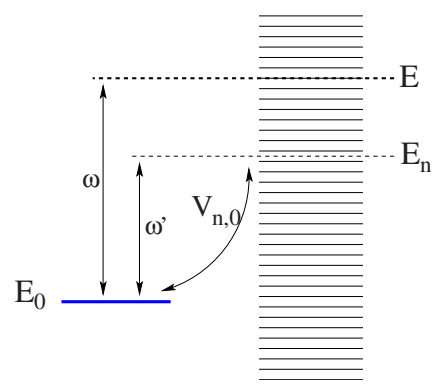

FIG. 2. (Color online) Illustration of the energy level scheme and the definition of $\omega$ and $\omega^{\prime}$, as explained in the main text.

The WM [8] features a perturbation matrix that does not discriminate between the levels and is given by a banded random matrix. For example,

$$
\mathcal{H}=\left[\begin{array}{cccccc}
E_{0} & V_{01} & V_{02} & 0 & 0 & 0 \\
V_{10} & E_{1} & V_{12} & V_{13} & 0 & 0 \\
V_{20} & V_{21} & E_{2} & V_{23} & V_{24} & 0 \\
0 & V_{31} & V_{32} & E_{3} & V_{34} & V_{35} \\
0 & 0 & V_{32} & V_{43} & E_{4} & V_{45} \\
0 & 0 & 0 & V_{43} & V_{54} & E_{5}
\end{array}\right] .
$$

Here we assume that all the levels are alike, and therefore Eq. (3) implies that

$$
\overline{\left|V_{n, m}\right|^{2}} \propto\left|E_{n}-E_{m}\right|^{s-1},
$$

where the overline indicates an RMT averaging over realizations, which from now on will be implicit in the analysis of the WM case. Unlike the FM case, here the dimension of the matrix $N$ has significance because any level can be reached by high order processes. As far as the theoretical analysis is concerned we assume the matrix to be of infinite size.

The assumed form (3) for the spectral function $\widetilde{C}(\omega)$ is motivated by the study of various model systems (e.g., those of Refs. [14-17]) where the assumption of "flat" band profile looks like an oversimplification. Thus it constitutes the natural generalization for the standard FM and WM. By integrating Eq. (3) over $\omega$ we see that the perturbation $V$ that appears in Eq. (2) is bounded provided $s>0$. The $s=1$ case is what we refer to as the Ohmic case, for which it is well known that both models leads to the same exponential decay for the survival probability [2]. For $s>2$ the effect of the continuum can be handled using first-order perturbation theory. We focus in the $0<s<2$ regime and consider the $s \neq 1$ case for which a nonlinear version of the Wigner decay problem is encountered.

We measure the energy taking $E_{0}$ as a reference and accordingly we use the notations $\omega \equiv E-E_{0}$ and $\omega_{n} \equiv E_{n}-E_{0}$. In the continuum limit any summation over $\omega_{n}$ is replaced by an integral over a variable $\omega^{\prime}$. See Fig. 2 for illustration.

In the numerical simulations we integrate the Schrödinger equation for the amplitudes $\psi_{n}(t)=\langle n \mid \psi(t)\rangle$ starting with the 
initial condition $\psi_{n}(0)=\delta_{n, 0}$. We use units such that $\hbar=1$, the density of states is $\varrho=1$ and $E_{0}=0$, and we assume a sharp bandwidth

$$
b=\varrho \omega_{c} \quad[\text { bandwidth }] .
$$

The matrix elements of $V$ are taken from a Gaussian distribution with zero mean and variance $\epsilon^{2}$ in the units as defined above. The integration is done using the self-expanding algorithm of [20] to eliminate finite-size effects, adding $10 \mathrm{~b}$ sites to each edge of the energy lattice whenever the probability of finding the "particle" at the edge sites exceeds $10^{-12}$.

\section{STRATEGY OF ANALYSIS}

We denote by $|n\rangle$ the unperturbed eigenstates of $\mathcal{H}_{0}$ and by $\left|E_{\nu}\right\rangle$ the perturbed eigenstates of $\mathcal{H}$. The local density of states LDOS with respect to the initial state $|0\rangle$ is defined as

$$
\rho(\omega)=\sum_{\nu}\left|\left\langle E_{\nu} \mid 0\right\rangle\right|^{2} \delta\left[\omega-\left(E_{\nu}-E_{0}\right)\right]
$$

Starting with the initial state the time-dependent state $|\psi(t)\rangle$ can be represented by the amplitudes $c_{n}(t)$, such that

$$
|\psi(t)\rangle=\sum_{n} c_{n}(t) e^{-i E_{n} t}|n\rangle .
$$

The probability distribution at time $t$ is

$$
P_{n}(t)=|\langle n|U(t)| 0\rangle|^{2}=\left|c_{n}(t)\right|^{2},
$$

where $U(t)=\exp [-i \mathcal{H} t]$ is the evolution operator. The survival probability is $P_{0}(t)$. We can associate with the energy distribution a probability density

$$
\rho_{t}(\omega)=\sum_{n} P_{n}(t) \delta\left(\omega-\omega_{n}\right) .
$$

The energy distribution is characterized by its dispersion

$$
\Delta E_{\text {sprd }}(t)=\left[\sum_{n}\left(E_{n}-E_{0}\right)^{2} P_{n}(t)\right]^{1 / 2}
$$

and by the median $E_{50 \%}=E_{0}$ and also by the $E_{25 \%}$ and $E_{75 \%}$ percentiles. The width of the core component is defined as

$$
\Delta E_{\text {core }}(t)=E_{75 \%}-E_{25 \%} .
$$

Our interest below is focused in $P_{0}(t)$, in $\Delta E_{\text {core }}(t)$, and in $\Delta E_{\text {sprd }}(t)$. We use the same measures in order to describe the LDOS distribution $\rho(\omega)$. See Fig. 3 for a cartoon that illustrates the significance of the different measures in the analysis.

The first stage of the analysis is to find the LDOS, also known as the strength function. In the FM case it can be done analytically using a standard Green's-function method. In the WM case it is possible to generalize the approach by Wigner and followers [8,9].

The survival amplitude is related to the LDOS. Namely, $c_{0}(t)$ can be written as the Fourier transform (FT) of $\rho(\omega)$. The derivation of this well-known fact is simple. Taking the energy reference as $E_{0}=0$ we have

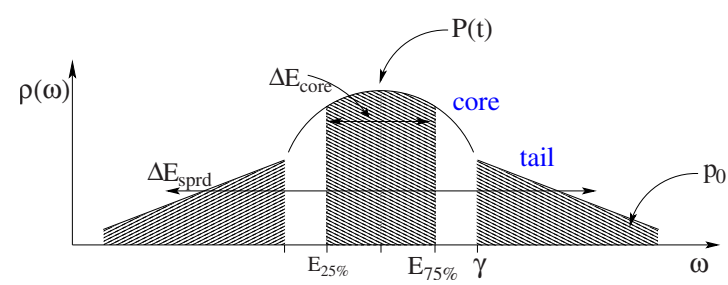

FIG. 3. (Color online) Cartoon that illustrates the structure of the energy distribution $\rho(\omega)$. The probability that would be held by the tails is $p_{0} \ll 1$ if FOPT strictly applied. It becomes of order unity (say 50\%) for a fully developed core-tail structure. The core has a semicircle line shape in the WM case, and its border $\gamma$ is determined self-consistently. The distribution is characterized by $P_{0}(t)$, by $\Delta E_{\text {core }}$, and by $\Delta E_{\text {sprd }}$ as explained in the main text.

$$
\begin{aligned}
c_{0}(t) & =\langle 0|U(t)| 0\rangle \\
& =\sum_{\nu}\left\langle 0\left|e^{-i \mathcal{H} t}\right| E_{\nu}\right\rangle\left\langle E_{\nu} \mid 0\right\rangle \\
& =\sum_{\nu}\left|\left\langle E_{\nu} \mid 0\right\rangle\right|^{2} e^{-i E_{\nu} t} \\
& =\sum_{\nu}\left|\left\langle E_{\nu} \mid 0\right\rangle\right|^{2} \mathrm{FT}\left[2 \pi \delta\left(\omega-E_{\nu}\right)\right] \\
& =\operatorname{FT}[2 \pi \rho(\omega)] .
\end{aligned}
$$

For obvious reasons there is resemblance between the saturation profile $\rho_{\infty}(\omega)$ and the $\operatorname{LDOS} \rho(\omega)$. In a stochastic (diagonal) approximation the former is the autoconvolution of the latter. But in order to find the time dependence of $\Delta E_{\text {core }}(t)$ and $\Delta E_{\text {sprd }}(t)$ it is not enough to know the LDOS. In order to obtain analytical results for the spreading we shall use a linear-response strategy that can be further refined in the FM case.

\section{TIME SCALES}

There are two frequency cutoffs, an infrared cutoff $\omega_{\varrho}$ $=\varrho^{-1}$ and an ultraviolet cutoff $\omega_{c}$, which are the mean level spacing and the range of the coupling, respectively. The associated time scales are the Heisenberg time $t_{\mathrm{H}}$ and the semiclassical time $t_{c}$ which are given by

$$
\begin{aligned}
& t_{\mathrm{H}}=2 \pi \varrho, \\
& t_{c}=2 \pi / \omega_{c} .
\end{aligned}
$$

We shall see that the continuum limit $\varrho^{-1} \rightarrow 0$ is well defined if $s>0$. If we further assume $s<2$ then well-defined results are obtained also in the universal limit $\omega_{c} \rightarrow \infty$. Thus in the range $0<s<2$ we should have in the continuum limit a cutoff free universal theory that constitutes a generalization of the Wigner decay problem. Note that for finite values of the cutoffs the actual range is more restricted as explained in Sec. V A and depicted in Fig. 4.

We shall see that the decay is characterized by what we call generalized Wigner time: 


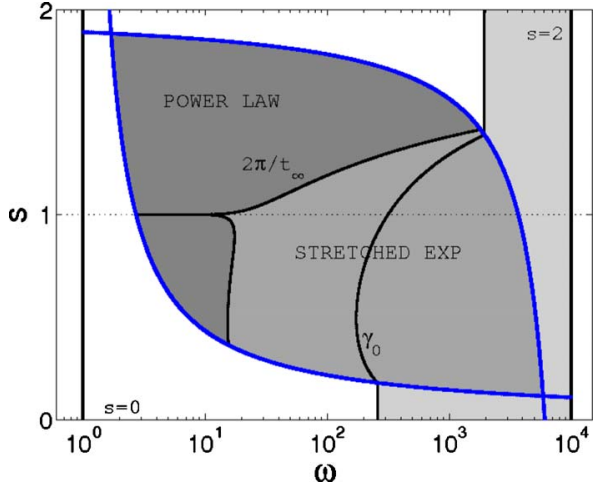

FIG. 4. (Color online) The different $\omega$ regions of the LDOS are plotted for $0 \leq s \leq 2$, relating to the (richer) FM case. The universal $\omega$ regions where the LDOS is cutoff free are shaded. The implied time-domain decay is indicated (note that upon the identification $t \sim 1 / \omega$ one can regard the horizontal axis as time stretching from right to left). The lower and upper cutoffs $\omega_{\varrho}$ and $\omega_{c}$ are indicated by vertical solid thick lines. The border of the different $\Delta(\omega)$ expressions written in Eq. (34) are plotted in curved solid (blue) lines. The curves of $\gamma_{0}$ and $2 \pi / t_{\infty}$ are plotted as well. The $\gamma_{0}$ curve was slightly modified for the purpose of presentation. The parameters used in this plot are $\omega_{\varrho}=1$ and $\omega_{c}=10^{4}$ and $\epsilon=2$.

$$
t_{0}=\left(\frac{2 \pi \epsilon^{2}}{\Gamma(3-s) \sin (s \pi / 2)}\right)^{-1 /(2-s)},
$$

where $\boldsymbol{\Gamma}$ is the gamma function. The numerical prefactor is explained and calculated in Sec. VI A. As far as order-ofmagnitude estimates are concerned a more practical expression is

$$
t_{0} \approx\left(\frac{2 \pi \epsilon^{2}}{(2-s) s}\right)^{-1 /(2-s)} .
$$

One observes clearly that $s \rightarrow 0$ and $s \rightarrow 2$ are limiting cases that require special attention due to the increased sensitivity to the infrared and to the ultraviolate cutoffs, respectively.

\section{LOCAL DENSITY OF STATES}

Following the presentation of $[14,20]$ we expect the LDOS to have in the most general case three regions: (i) the core that consists of the levels that are mixed nonperturbatively; (ii) the first-order tails; and (iii) the higher-order tails. We now explain this terminology and the associated phenomenology.

The LDOS is determined by the overlaps $\left\langle E_{\nu} \mid 0\right\rangle$ of the initial state with the perturbed eigenstates. So naturally the first question that arises is whether we can use perturbation theory for the calculation. First-order perturbation theory (FOPT) assumes that we can associate perturbed and unperturbed levels (no mixing), hence $\left\langle E_{\nu=0} \mid n=0\right\rangle \sim \mathcal{O}(1)$. If this is indeed the case we say that the core consists of one level only, while the tails consist of all the $\left\langle E_{\nu \neq 0} \mid n=0\right\rangle$ overlaps. Typically FOPT gives a leading order approximation for the tails, while higher orders are essential for those levels that are not coupled directly [10]. In our model the higher order tails reside at $\omega>\omega_{c}$ out of the range of physical interest because $\omega_{c}$ is assumed to be very large.
The first-order tails are fully determined by the spectral function $\widetilde{C}(\omega)$. The self consistent condition for the validity of FOPT is $p_{0} \ll 1$, where $p_{0}$ is defined as the probability which is carried by the tails:

$$
p_{0}=\sum_{n}\left|\frac{V_{n 0}}{E_{n}-E_{0}}\right|^{2}=\int \frac{\tilde{C}(\omega)}{\omega^{2}} \frac{d \omega}{2 \pi} .
$$

By substituting the spectral function of Eq. (3) we get

$$
p_{0}=\epsilon^{2} \begin{cases}2 \boldsymbol{\Gamma}(s-2) \omega_{c}^{s-2} & \text { for } s>2 \\ (2-s)^{-1} \varrho^{2-s} & \text { for } s<2 .\end{cases}
$$

In the continuum limit $p_{0}$ is infrared divergent if $s<2$, and consequently FOPT does not apply even if $\epsilon$ is made very small. This is the case of our interest, and it should be contrasted with the $s>2$ case for which FOPT applies in the weak-coupling limit. In the latter case the LDOS can be written schematically as

$$
\rho(\omega)=\left(1-p_{0}\right) \delta(\omega)+p_{0} \frac{1}{\omega_{c}} \tilde{f}\left(\frac{\omega}{\omega_{c}}\right),
$$

where the second term stands for $\widetilde{C}(\omega) /\left(2 \pi \omega^{2}\right)$. The natural question that arises is what happens to this line shape in the $s<2$ case and in particular whether there are remnants of FOPT. It turns out that generically the answer is positive [21]. In spite of the mixing of nearby levels, the tails are still given by the FOPT expression. Accordingly we can identify in the LDOS a core region $|\omega|<\gamma_{0}$ that contains the large overlaps, and FOPT tails that dominate the outer $|\omega|>\gamma_{0}$ regions. The characteristic frequency can be determined either analytically or self-consistently and consequently the generalized Wigner time is identified as $t_{0}=1 / \gamma_{0}$. This phenomenological picture will be formulated in a more precise way in the subsequent subsections.

We are going to analyze the LDOS for $s<2$, where we have to go beyond FOPT, but still can use the core-tail phenomenology. The nonperturbative core within $|\omega|<\gamma_{0}$ has nonuniversal structure that depends on the details of the model. There are various strategies to deduce the structure of the core. In particular: the Green's-function method that we are going to use in the FM case; the RMT calculation of the moments as in the pioneering work of Wigner [8,9]; and the semiclassical reasoning if the model has a classical limit [14-16,22].

A few words about the Lamb shift are in order. FOPT allows us to calculate the shift of $E_{0}$ due to the repulsion by the other levels:

$$
\Delta(0)=\sum_{n} \frac{\left|V_{n, 0}\right|^{2}}{E_{0}-E_{n}}=-\int_{-\omega_{c}}^{+\omega_{c}} \frac{\widetilde{C}\left(\omega^{\prime}\right)}{\omega^{\prime}} \frac{d \omega^{\prime}}{2 \pi} .
$$

If $s>0$ the Lamb shift is not infrared divergent provided we keep away from the energy floor, and we get $\Delta(0)=0$ due to the symmetry of $\widetilde{C}(\omega)$. This of course does not mean that Lamb-shift physics is irrelevant. In the analysis of the FM model we shall define a spectral function $\Delta(\omega)$ that plays a major role in the analysis. 


\section{A. LDOS: FM}

In the FM case it is possible to derive an exact expression for the LDOS either via the Green's-function formalism (Appendix A) or from a straightforward elementary calculation (Appendix B). The final result is

$$
\rho(\omega)=\frac{1}{\pi} \frac{\Gamma(\omega) / 2}{[\omega-\Delta(\omega)]^{2}+[\Gamma(\omega) / 2]^{2}},
$$

where

$$
\begin{aligned}
& \Gamma(\omega)=\sum_{n}\left|V_{n, 0}\right|^{2} 2 \pi \delta\left(E-E_{n}\right)=\widetilde{C}(\omega), \\
& \Delta(\omega)=\sum_{n} \frac{\left|V_{n, 0}\right|^{2}}{E-E_{n}}=\int_{-\infty}^{+\infty} \frac{\widetilde{C}\left(\omega^{\prime}\right)}{\omega-\omega^{\prime}} \frac{d \omega^{\prime}}{2 \pi} .
\end{aligned}
$$

In the Ohmic case $(s=1)$ we have $\Delta(\omega)=0$ and $\Gamma(\omega)$ =const, and consequently the LDOS is the familiar Lorentzian. In order to calculate $\Delta(\omega)$ for $s \neq 1$ we exploit the fact that $\widetilde{C}(\omega)$ is an even function, while $\Delta(\omega)$ comes out odd. Consequently we can write Eq. (28) as

$$
\begin{aligned}
\Delta(\omega) & =\epsilon^{2} \frac{\omega}{\pi} \int_{\omega_{\varrho}}^{\omega_{c}} \frac{\left(\omega^{\prime}\right)^{s-1} d \omega^{\prime}}{\omega^{2}-\omega^{\prime 2}} \\
& =-\epsilon^{2}|\omega|^{s-1} \operatorname{sgn}(\omega) \int_{\ln |\omega / \omega|}^{\ln \left|\omega_{c} / \omega\right|} \frac{e^{(s-1) x}}{\sinh (x)} d x,
\end{aligned}
$$

where $\omega_{\varrho}=\varrho^{-1}$ is the level spacing, and we used the substitution $\omega^{\prime}=\omega e^{x}$. For $0<s<2$ it is possible to take the limits $\omega_{\varrho} \rightarrow 0$ and $\omega_{c} \rightarrow \infty$. Using the integral

$$
\int_{-\infty}^{+\infty} \frac{\sinh [(s-1) x]}{\sinh (x)} d x=-\pi \cot (s \pi / 2),
$$

we get the cutoff-free result

$$
\Delta(\omega)=\epsilon^{2} \pi \cot (s \pi / 2)|\omega|^{s-1} \operatorname{sgn}(\omega)
$$

In contrast to that the marginal cases $s=0$ and $s=2$ are lower and upper cut-off dependent, respectively,

$$
\begin{gathered}
\Delta(\omega)=-\epsilon^{2} \omega \ln \left|1-\left(\omega_{c} / \omega\right)^{2}\right|, \quad s=2, \\
\Delta(\omega)=\epsilon^{2} \frac{1}{\omega} \ln \left|\left(\omega / \omega_{\varrho}\right)^{2}-1\right|, \quad s=0 .
\end{gathered}
$$

The crossover from the " $0<s<1$ " to the " $s=0$ " and the " $s$ $=2$ " expressions is not sharp. Looking carefully at the integral we see that the condition for a cut-off independent result is (see Fig. 4)

$$
\omega_{\varrho} e^{1 / s} \ll \omega \ll \omega_{c} e^{-1 /(2-s)} .
$$

Having obtained $\Delta(\omega)$ we can substitute it into Eq. (26) and get the LDOS. Both $\Gamma$ and $\Delta$ are $\propto \omega^{s-1}$, with $s$-dependent prefactors. One can define a characteristic crossover frequency $\gamma_{0}$ above which the $\omega^{2}$ term in the denominator if Eq. (26) dominates. In the universal regime this leads to

$$
\gamma_{0} \approx\left(\frac{\epsilon^{2}}{|\sin (s \pi / 2)|}\right)^{1 /(2-s)},
$$

while in the limiting cases it attains the values

$$
\begin{aligned}
& \gamma_{0} \approx \omega_{c} e^{-1 / 2 \epsilon^{2}} \quad \text { as } s \rightarrow 2, \\
& \gamma_{0} \approx \epsilon \ln (\varrho \epsilon) \text { as } s \rightarrow 0 .
\end{aligned}
$$

Depending whether $\omega \ll \gamma_{0}$ or $\omega \gg \gamma_{0}$ one obtains different approximations for the LDOS that can be packed together in the following writing style:

$$
\rho(\omega)=\frac{1}{\pi}\left\{\frac{\Gamma / 2}{\Delta^{2}+(\Gamma / 2)^{2}}, \frac{\Gamma / 2}{\omega^{2}}\right\} .
$$

Consequently we get for $0<s<2$

$$
\rho(\omega)=\left\{\left(\frac{\sin (s \pi / 2)}{\pi \epsilon}\right)^{2} \frac{1}{|\omega|^{s-1}}, \frac{\epsilon^{2}}{|\omega|^{3-s}}\right\} .
$$

This validity regions of the expression in the curly brackets are illustrated in Fig. 4. After FT they are reflected in time domain as implied by the analysis in Fig. 5, which we further discuss later. The numerically determined LDOS in the FM case is displayed in Fig. 6 and contrasted with the WM case.

Equation (39) holds provided $\gamma_{0}$ is in the range that has been specified in Eq. (34). This constitutes a condition on $\epsilon$ that can be regarded as a generalized version of the FGR condition. For marginal values of $s$ it becomes more difficult to satisfy the generalized FGR condition, and eventually the range of the universal behavior as defined in Eq. (34) shrinks to zero [see Fig. 4]. In particular for $s \rightarrow 2$, once the generalized FGR condition breaks down, we get

$$
\rho(\omega)=\left\{\frac{1}{4 \epsilon^{2}} \frac{1}{|\omega| \ln ^{2}\left|\omega_{c} / \omega\right|}, \frac{\epsilon^{2}}{|\omega|}\right\} .
$$

In this expression the $1 / \omega$ tails prevail for $\omega>\gamma_{0}$, analogous to Eq. (39), but here $\gamma_{0}$ is $\omega_{c}$ dependent.

\section{B. LDOS: WM}

As discussed in the beginning of this section, for $s>2$ we can calculate the LDOS using FOPT. In contrast, for $s<2$, FOPT breaks down for any finite coupling, because in the continuum limit $p_{0}$ is infrared divergent. Still we can use the FOPT result down to a frequency $\gamma_{o}$ which we estimate below in a self consistent manner. For lower frequencies the LDOS is dominated by a nonperturbative core which is model specific and in that sense nonuniversal. For the WM we write schematically

$$
\rho(\omega) \approx\left\{\text { Semicircle, } \frac{\epsilon^{2}}{|\omega|^{3-s}}\right\} .
$$

This functional form is illustrated in Fig. 3 and tested numerically in Fig. 6. The reasoning behind this expression is further detailed below.

The crossover frequency $\gamma_{o}$ is almost the same as $\gamma_{0}$ which we define in the time domain analysis of the next 

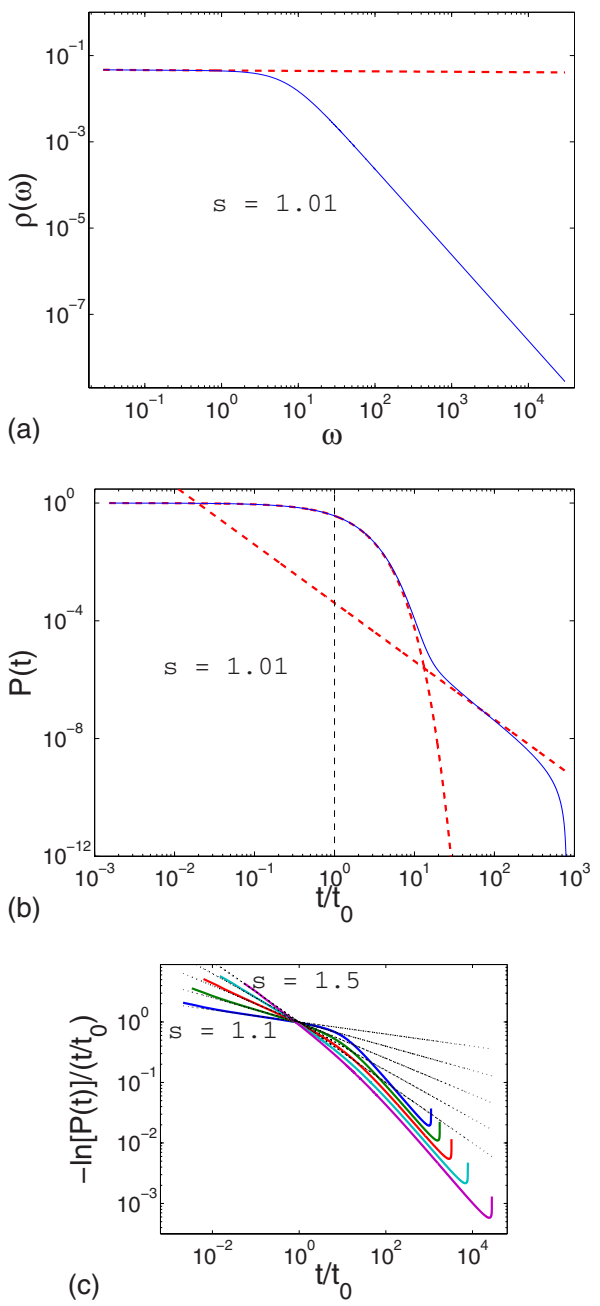

FIG. 5. (Color online) Upper panel: the FM analytical LDOS for $s=1.01$. The core power-law [Eq. (39)] is indicated by a dashed red line. Middle panel: the survival probability $P_{0}(t)$ computed using numerical FT of the LDOS. The exponential-like decay [based on Eq. (49)] and the power-law decay [based on Eq. (50)] are plotted in dashed red lines. The vertical dashed black line emphasis the fact that the crossover time $t_{\infty}$ is different from $t_{0}$. Lower panel: the same for various values of $s \in[1.1,1.5]$. For short times there is a good agreement with an exponential-like decay [based on Eq. (49)], which is plotted in dotted black lines.

section [the distinction between the two is clarified after Eq. (55). We define the crossover frequency $\gamma_{o}$ by the equation

$$
\int_{|\omega|>\gamma_{o}} \frac{\widetilde{C}(\omega)}{\omega^{2}} \frac{d \omega}{2 \pi} \sim 50 \%,
$$

which gives

$$
\gamma_{o} \approx\left(\frac{\epsilon^{2}}{2-s}\right)^{1 /(2-s)} .
$$

The FOPT tails are dominated by $\mathcal{H}_{0}$, while $V$ is regarded as a perturbation. In contrast to that the core is fully dominated by $V$. Following Ref. [9] we can argued that the core of the LDOS is a semicircle of width $\Delta E_{\mathrm{sc}}$, which in our case is given by the expression

$$
\Delta E_{\mathrm{sc}}=\left[\int_{0}^{\gamma_{o}} \tilde{C}(\omega) \frac{d \omega}{2 \pi}\right]^{1 / 2} .
$$

In this expression we use the effective bandwidth $\gamma_{o}$ rather than the real bandwidth $\omega_{c}$ as implied by our core-tail hypothesis. Performing the integration we get

$$
\Delta E_{\mathrm{sc}} \sim \gamma_{o},
$$

which implies that the calculation is indeed self-consistent, i.e., there is a well-defined frequency $\gamma_{o}$ at which the coretail crossover takes place.

\section{DECAY OF THE SURVIVAL PROBABILITY}

We turn now to the study of wave-packet dynamics, with the objective to contrast the results of the FM with those of the generalized WM. We assume that the initial preparation is deltalike in energy space. The temporal behavior of the wave packet, is characterized by three quantities: (a) the survival probability is directly related to the LDOS analysis; (b) the core width reflects the nonuniversal component of the wave packet; (c) the energy spreading reflects the tails of the wave packet.

In the present section we study the survival amplitude, which is obtained via FT of the LDOS, namely, $c_{0}(t)$ $=\mathrm{FT}[2 \pi \rho(\omega)]$. For $s>2$ the FT of the FOPT estimate Eq. (24) has a simple crossover at $t_{c}$ to saturation

$$
c_{0}(t)=\left(1-p_{0}\right)+p_{0} f\left(t / t_{c}\right) \sim 1-p_{0}\left[1-\frac{1}{\left(t / t_{c}\right)^{s-2}}\right] .
$$

For $s<2$ the core is not a discrete delta function but rather has a width $\gamma_{o}$ which implies a progressive decay on time scale $1 / \gamma_{o}$. Our focus below is on the regime $0<s<2$, and we pay some extra attention to the limiting cases $s \rightarrow 0$ and $s \rightarrow 2$.

\section{A. Survival probability: WM}

In the WM case the LDOS has power-law tails $1 /|\omega|^{1+\alpha}$ with $\alpha=2-s$ and a smooth semicircle core. Therefore its FT is exponential-like for all times. The similarity to the $\alpha$-stable Levy distribution suggests a stretched exponential decay which is confirmed by our simulations. There is an optional argument that supports a stretched exponential behavior. The stretched exponential can be regarded as the solution of the following phenomenological rate equation for the survival probability:

$$
\dot{\mathcal{P}}(t)=-\Gamma(\mathrm{const} / t) P_{0}(t) .
$$

This phenomenological equation involves a time dependent effective decay rate into the quasi continuum. It is estimated as $\Gamma(\omega)$ with $\omega \sim 1 / t$ which reflects the energy uncertainty at time $t$. This phenomenological approach does not provide a definite result for the numerical constant. A reasonable procedure is to determine this constant from the short time behavior, which is determined by the tails. We recall the following FT relation: 

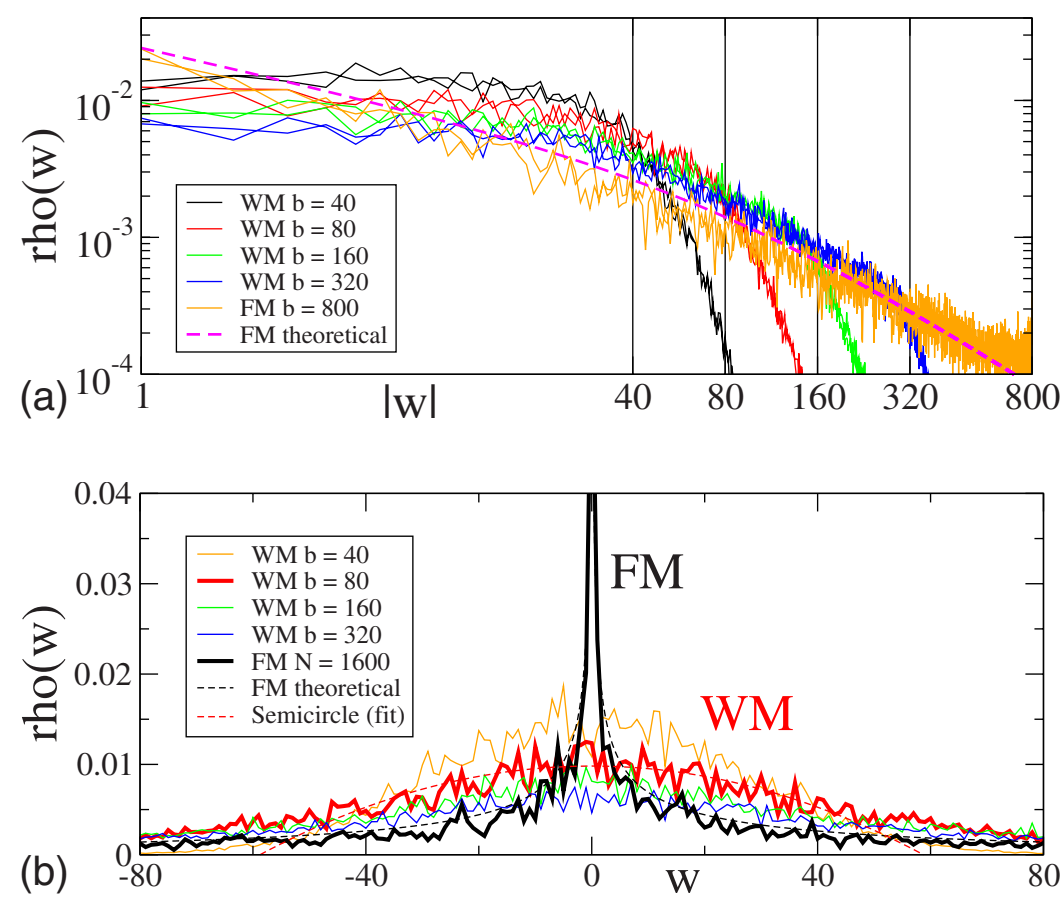

where $\beta>0$. Switching the roles of $t$ and $\omega$, the inverse FT implies that power-law tails $1 /|\omega|^{1+\alpha}$ are related to discontinuity or singularity $C|t|^{\alpha}$ at time domain, where $C=-[2 \Gamma(1$ $+\alpha) \sin (\alpha \pi / 2)]^{-1}$. This leads to the result

$$
c_{0}(t)=\exp \left[-\frac{1}{2}\left(\frac{t}{t_{0}}\right)^{2-s}\right] \text {, }
$$

where $t_{0}$ is defined as in Eq. (20). Going back to the phenomenological rate equation it implies that the numerical constant is $\{(2-s) /[\boldsymbol{\Gamma}(3-s) \sin (s \pi / 2)]\}^{1 /(s-1)}$.

\section{B. Survival probability: FM}

In the FM case the core is singular and therefore a power law takes over for $t>t_{\infty}$, where the crossover time $t_{\infty}$ is determined below. In Fig. 5 we present numerical FTs that support this statement. Note that in the $s=2$ limit the power law becomes a logarithmic decay.

In order to obtain the explicit expression for the powerlaw decay we use the FT relation of Eq. (48). Due to the discontinuity or singularity at $\omega=0$ we get for large times

$$
\begin{aligned}
c_{0}(t) & =\frac{\sin [(s-1) \pi] \sin (s \pi / 2) \Gamma(2-s)}{\pi^{2} \epsilon^{2} t^{2-s}} \\
& =\frac{2 \sin [(s-1) \pi]}{(2-s) \pi}\left(\frac{t_{0}}{t}\right)^{2-s},
\end{aligned}
$$

where in the last equation we expressed $c_{0}(t)$ by $t_{0}$. Comparing the exponential-like and the power law we find an expression for the crossover time
FIG. 6. (Color online) The LDOS for the FM and for the WM via direct diagonalization of $1600 \times 1600$ matrices with $s=1.5$ and $\epsilon=1.44$. The units are such that $\varrho=1$, and hence the bandwidth is $\omega_{c}=b$. In the FM case formally $b=N / 2$. Upper panel: the log-log scale emphasizes the emergent universality of the tails as the cutoff $\omega_{c}$ is taken to infinity. Lower panel: the logarithmiclinear scale emphasizes the difference in the nonuniversal core component: FM has a singular core, while WM has a smooth semicirclelike core.

$$
t_{\infty}=\left|2 \ln \left(\frac{2 \sin (|s-1| \pi)}{(2-s) \pi}\right)\right| t_{0}^{1 /(2-s)} .
$$

This expression diverges in the limit $s=1$, implying that only the exponential survives. The numerical simulations presented in Figs. 7 and 8 support our findings.

The marginal case $s=2$ requires special treatment. In this case the strength of the interaction $(\epsilon)$ is a dimensionless parameter. The $\propto 1 / \omega$ tail of the LDOS in Eq. (40) is $\omega_{c}$ independent but with the $\omega_{c}$ dependent lower cutoff of Eq. (36). For the purpose of FT we approximate the LDOS as

$$
\rho(\omega) \approx \frac{\epsilon^{2}}{\sqrt{\omega^{2}+\left(1 / t_{0}\right)^{2}}}
$$

and from the integral representation of the modified Bessel function of the second kind, using the approximation $K_{0}(\tau)$ $\approx$ const $-\ln (\tau)$ it follows that

$$
c_{0}(t) \approx \frac{\epsilon^{2}}{\pi} \log \left(\frac{t_{0}}{t}\right) \text { for } t_{c} \ll t \ll t_{0} .
$$

In order to clarify how the $s=2$ case is related to the general $s$ expression, it is useful to note that for very small $\beta$ one has $t^{\beta} \sim \beta \ln (t)$. It is also important to realize that for $t<t_{0}$ both the $s=1$ exponential and the $s=2$ logarithm are consistent with the short time $1-\left(t / t_{0}\right)^{2-s}$ expression that holds for general $s$. In contrast to that the long time decay is sensitive to the small frequency features of the LDOS.

\section{EVOLUTION OF THE CORE WIDTH}

In this section we study the time evolution of the core as reflected in the width of the energy distribution. The width $\Delta E_{\text {core }}(t)$ is intimately related to $P_{0}(t)$. It starts to rise at $t$ $\sim t_{0}$ when $P_{0}(t)=50 \%$, and its saturation value is determined by the core width of the LDOS $\sim \gamma_{0}$. Thus $\Delta E_{\text {core }}$ should 

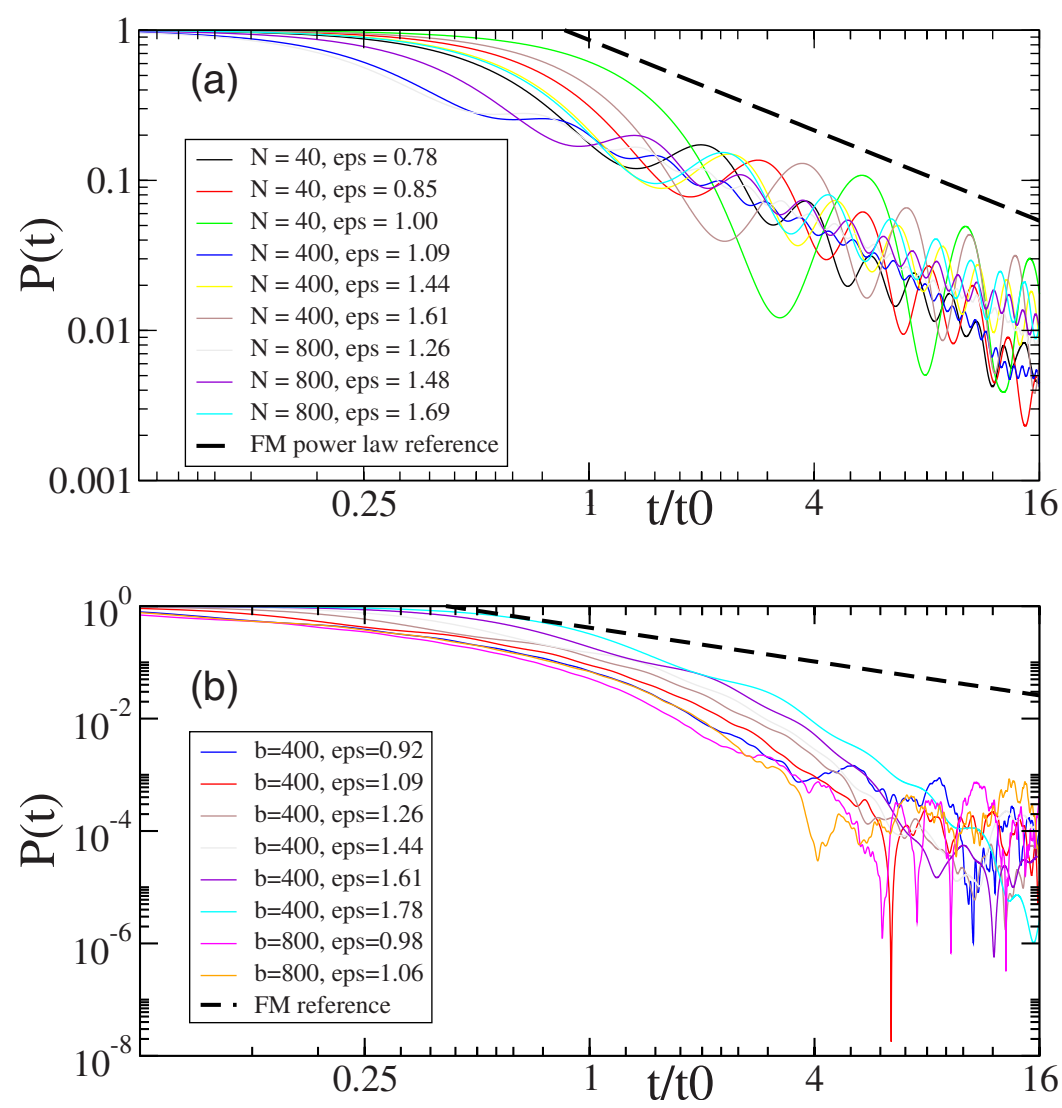

FIG. 7. (Color online) The survival probability $P_{0}(t)$ for the FM (top) and for the WM (bottom), as a function of $t / t_{0}$, in log-log scale, for various values of $\varepsilon$. The predicted FM power law is presented as a dashed line. For all curves $\varrho=1$ and $s=1.5$. exhibit one parameter scaling with respect to $t_{0}$. This has been verified numerically, and is shown in Fig. 9. A more elaborated analysis follows below.

For $t \ll t_{0}$ the initial state carries most of the probability, and therefore we can use FOPT to obtain in analogy with Eq. (24):

$$
\rho_{t}(\omega)=\delta(\omega)+\frac{1}{2 \pi} \frac{\widetilde{C}(\omega)}{\omega^{2}}[2 \sin (\omega t / 2)]^{2}
$$

with normalization measure $d \omega$. As long as this expression holds we say that the core component contains one level only. It is clear that after a longer time $\rho_{t}(\omega)$ becomes similar to the $\operatorname{LDOS} \rho(\omega)$.

Accordingly it is natural to ask what is the time evolution of the core-tail structure. In particular one would like to estimate the time dependence of the border $\gamma(t)$ between the core and the tail. Using the same reasoning as in Eq. (42) we get for $\gamma(t)$ the equation

$$
\epsilon^{2} \times\left[\frac{1}{s}\left(t^{2-s}-\gamma^{s} t^{2}\right)+\frac{1}{2-s}\left(t^{2-s}\right)\right] \sim 50 \% .
$$

From this equation it follows that there is a sharp crossover from $\gamma(t)=\varrho^{-1}$ to $\gamma(t) \sim \gamma_{o}$ during the time interval $\left[t_{0}, t_{o}\right]$, where $t_{0}$ is given by Eq. (21) and $t_{o}=1 / \gamma_{o}$ is given by Eq. (43). In Fig. 9 we present the results of the numerical analysis. Our data, indicate that the expected one-parameter scaling is obeyed. We have verified that the deviations from the expected $\epsilon$ dependence (for large $\epsilon$ ) are an artifact due to having finite (rather then infinite) bandwidth.

\section{TIME-DEPENDENT SPREADING}

In this section we analyze a very different characteristic of the evolving wave packet, which is the spreading $\Delta E_{\text {sprd }}(t)$. Unlike the width which is determined by the core, the spreading is determined by the tails of the distribution. Irrespective of whether we deal with FM or with WM the tails are determined by FOPT and therefore the naive expectation is to have a $t_{c}$ dependent rather than $t_{0}$-dependent evolution. We shall see that this is roughly but not quite correct.

For the analysis one can use the traditional strategy of Refs. [16,20,23], leading to the following spreading formula (see Appendix C):

$$
\left[\Delta E_{\mathrm{sprd}}(t)\right]^{2}=C(0,0)+C(t, t)-C(0, t)-C(t, 0),
$$

where

$$
C\left(t^{\prime}, t^{\prime \prime}\right)=\left\langle V\left(t^{\prime}\right) V\left(t^{\prime \prime}\right)\right\rangle \text {. }
$$

This expression is formally exact. But in order to get a practical expression the LRT approximation assumes that it is possible to calculate the correlation function with the unperturbed Hamiltonian. Accordingly $C\left(t^{\prime}, t^{\prime \prime}\right) \approx C\left(t^{\prime}-t^{\prime \prime}\right)$, where $C(t)$ is the inverse FT of $\widetilde{C}(\omega)$, leading to

$$
\Delta E_{\text {sprd }}(t)=\{2[C(0)-C(t)]\}^{1 / 2} .
$$

This expression implies that 

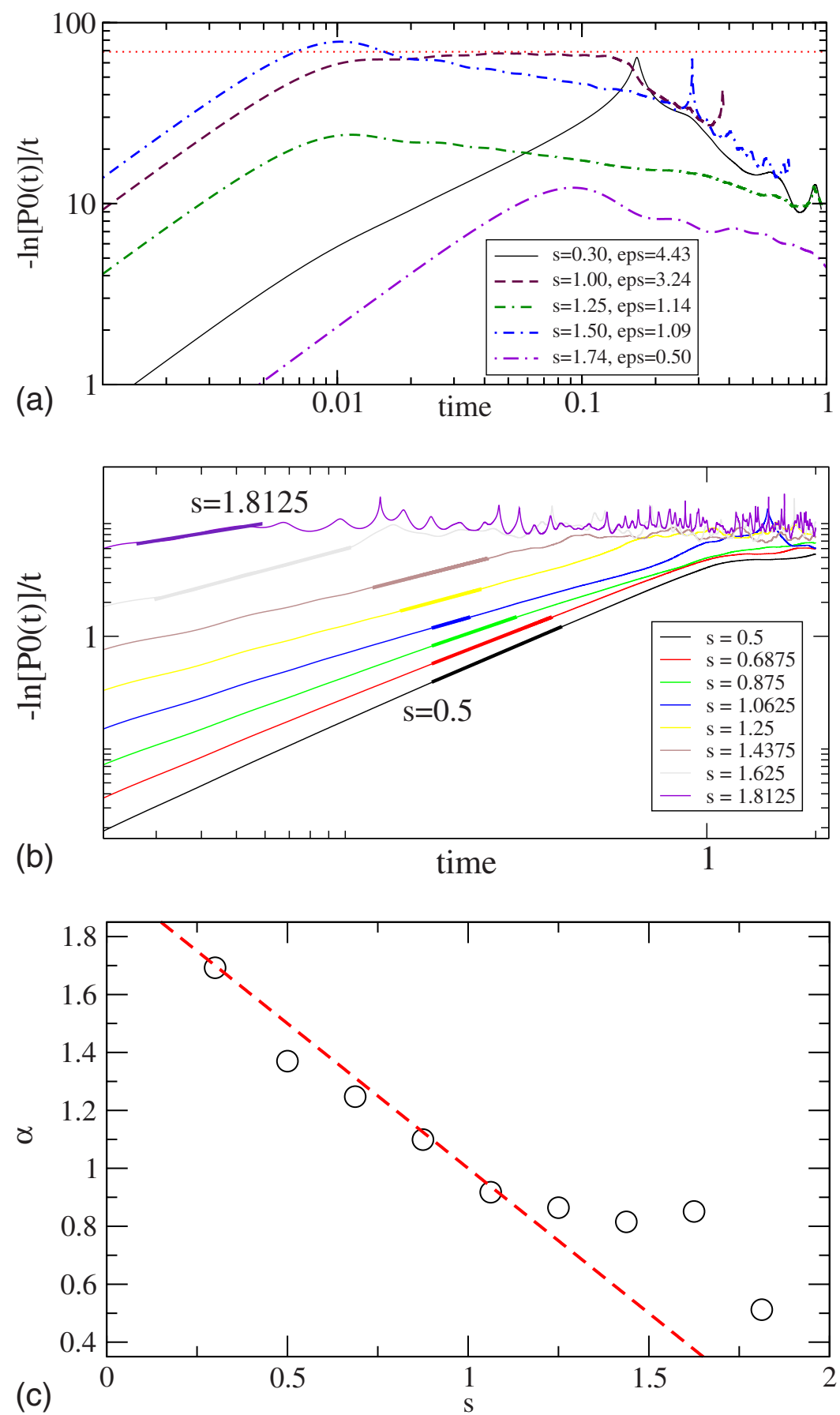

This saturation values, which diverges in the $\omega_{c} \rightarrow \infty$ limit, is attained after a short transient time $t_{c} \rightarrow 0$, while the generalized Wigner time $t_{0}$ is not reflected. The above expressions agrees with the numerical calculations, as shown in Fig. 10.

The LRT approximation $C\left(t^{\prime}, t^{\prime \prime}\right) \approx C\left(t^{\prime}-t^{\prime \prime}\right)$ makes sense if the correlation function is stationary. This is not the case with FM. By inspection a more appropriate approximation in the latter case should take into account the decay of the initial state and hence it should incorporate the survival amplitude $c_{0}(t)$ as follows:

FIG. 8. (Color online) Upper panel: a replot of Fig. 7 using $Y=-\ln \left[P_{0}(t)\right] / t$ and $X=t$ in a log$\log$ scale, for representative runs, showing that the decay in the WM case is described by a stretched exponential. The red bold dashed line has zero slope, corresponding to simple exponential decay for $s=1$. Middle panel: additional curves for various values of $s$ are plotted. The highlighted (thickened) segments demonstrate exponential-like decay. Lower panel: the power $\alpha$ is extracted by fitting to the form $P_{0}(t)$ $=\exp \left[-t^{\alpha}\right]$ of the highlighted segments of the middle panel. The expected power $\alpha=2-s$ is indicated by a dashed red line.
Within the framework of this approximation we get

$$
\Delta E_{\mathrm{sprd}}(t) \approx\left[\left(1+P_{0}(t)\right) C(0)-2 c_{0}(t) C(t)\right]^{1 / 2},
$$

where $P_{0}(t)=\left|c_{0}(t)\right|^{2}$. This leads to a saturation value smaller by factor $\sqrt{2}$ compared with the WM case, reflecting the nonstationary decay of the fluctuations as a function of time. More interestingly Eq. (61) contains a cutoff independent term that reflects the universal time scale $t_{0}$.

In fact in the FM case it is possible to obtain an exact result. First we notice that by definition $c_{0}(t)=\left\langle e^{-i \mathcal{H} t}\right\rangle$, where the expectation value here and below is with respect 

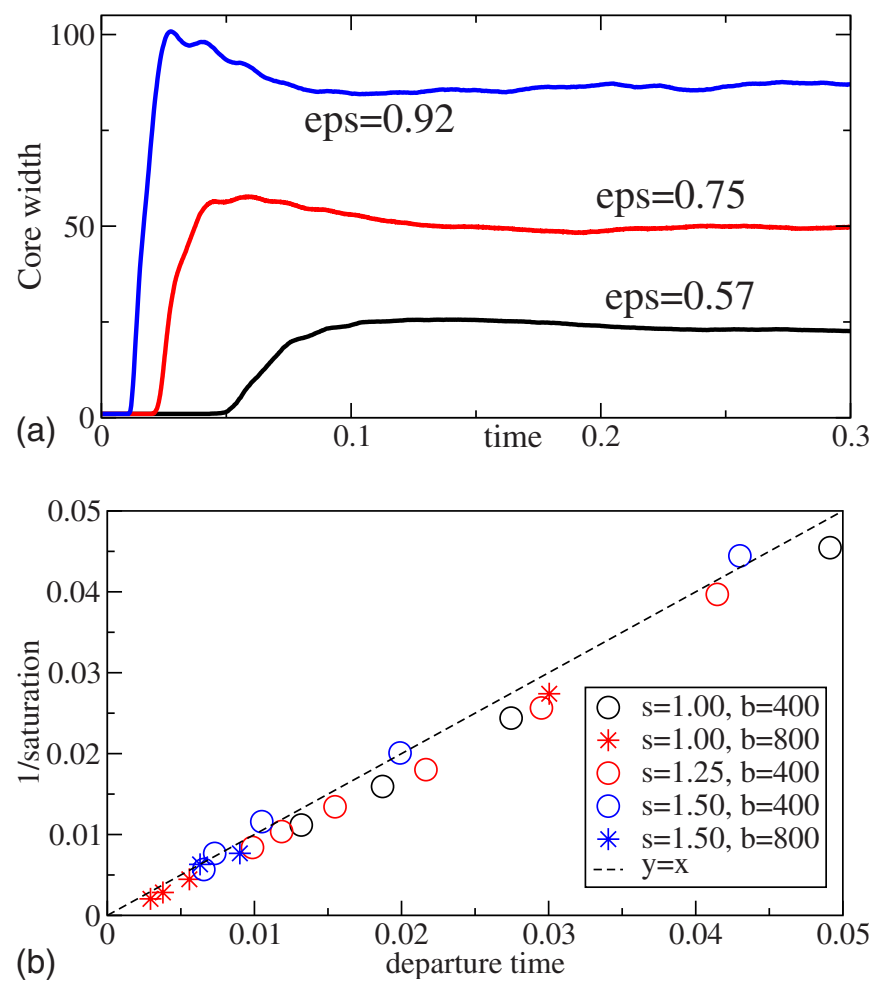

FIG. 9. (Color online) Upper panel: examples for the time evolution of $\Delta E_{\text {core }}$ for $s=1.5$ and $b=800$ in the WM case. Lower panel: the extracted departure time versus the extracted inverse saturation value. This scatter diagram demonstrates the validity of one parameter scaling.

to the initial $|0\rangle$ state. By differentiating we get either $\dot{c}_{0}(t)=-i\left\langle\mathcal{H} e^{-i \mathcal{H} t}\right\rangle$ or $\dot{c}_{0}(t)=-i\left\langle e^{-i \mathcal{H} t} \mathcal{H}\right\rangle$. Setting $\mathcal{H}=\mathcal{H}_{0}+V$ and using the convention $E_{0}=0$ for the $|0\rangle$ eigenstate of $\mathcal{H}_{0}$, we find

$$
\dot{c}_{0}(t)=-i\left\langle V e^{-i \mathcal{H} t}\right\rangle=-i\left\langle e^{-i \mathcal{H} t} V\right\rangle
$$

Similarly we can handle the second derivative leading to

$$
\ddot{c}_{0}(t)=-\left\langle\mathcal{H} e^{-i \mathcal{H} t} \mathcal{H}\right\rangle=-\left\langle V e^{-i \mathcal{H} t} V\right\rangle .
$$

We now realize that the correlations in Eq. (56) can be expressed using $C(t)$ and $c_{0}(t)$. Trivially we have

$$
\langle V(0) V(0)\rangle=\left\langle V^{2}\right\rangle=C(0) .
$$

In the FM model the only nonzero elements of $V$ are $V_{0, n}$ and $V_{n, 0}$ with $n \neq 0$. Consequently we can factorize also the other correlations as follows:

$$
\begin{aligned}
& \langle V(t) V(t)\rangle=\left\langle e^{i \mathcal{H} t}\right\rangle\left\langle V^{2}\right\rangle\left\langle e^{-i \mathcal{H} t}\right\rangle+\left\langle e^{i \mathcal{H} t} V\right\rangle\left\langle V e^{-i \mathcal{H} t}\right\rangle, \\
& \langle V(0) V(t)\rangle=\left\langle V e^{i \mathcal{H} t}\right\rangle\left\langle V e^{-i \mathcal{H} t}\right\rangle+\left\langle V e^{i \mathcal{H} t} V\right\rangle\left\langle e^{-i \mathcal{H} t}\right\rangle, \\
& \langle V(t) V(0)\rangle=\left\langle e^{i \mathcal{H} t} V\right\rangle\left\langle e^{-i \mathcal{H} t} V\right\rangle+\left\langle e^{i \mathcal{H} t}\right\rangle\left\langle V e^{-i \mathcal{H} t} V\right\rangle .
\end{aligned}
$$

It follows that

$$
\begin{gathered}
C(0,0)=C(0), \\
C(t, t)=P_{0}(t) C(0)+\dot{c}_{0}(t)^{2}, \\
C(0, t)=C(t, 0)=\dot{c}_{0}(t)^{2}-c_{0}(t) \ddot{c}_{0}(t) .
\end{gathered}
$$

Substituting into Eq. (56) we get the result

$$
\Delta E_{\mathrm{sprd}}(t)=\left[\left(1+c_{0}^{2}(t)\right) C(0)-\dot{c}_{0}(t)^{2}+2 c_{0}(t) \ddot{c}_{0}(t)\right]^{1 / 2} .
$$

We note that for short times $\left(t \ll t_{0}\right)$ we can use the approximations

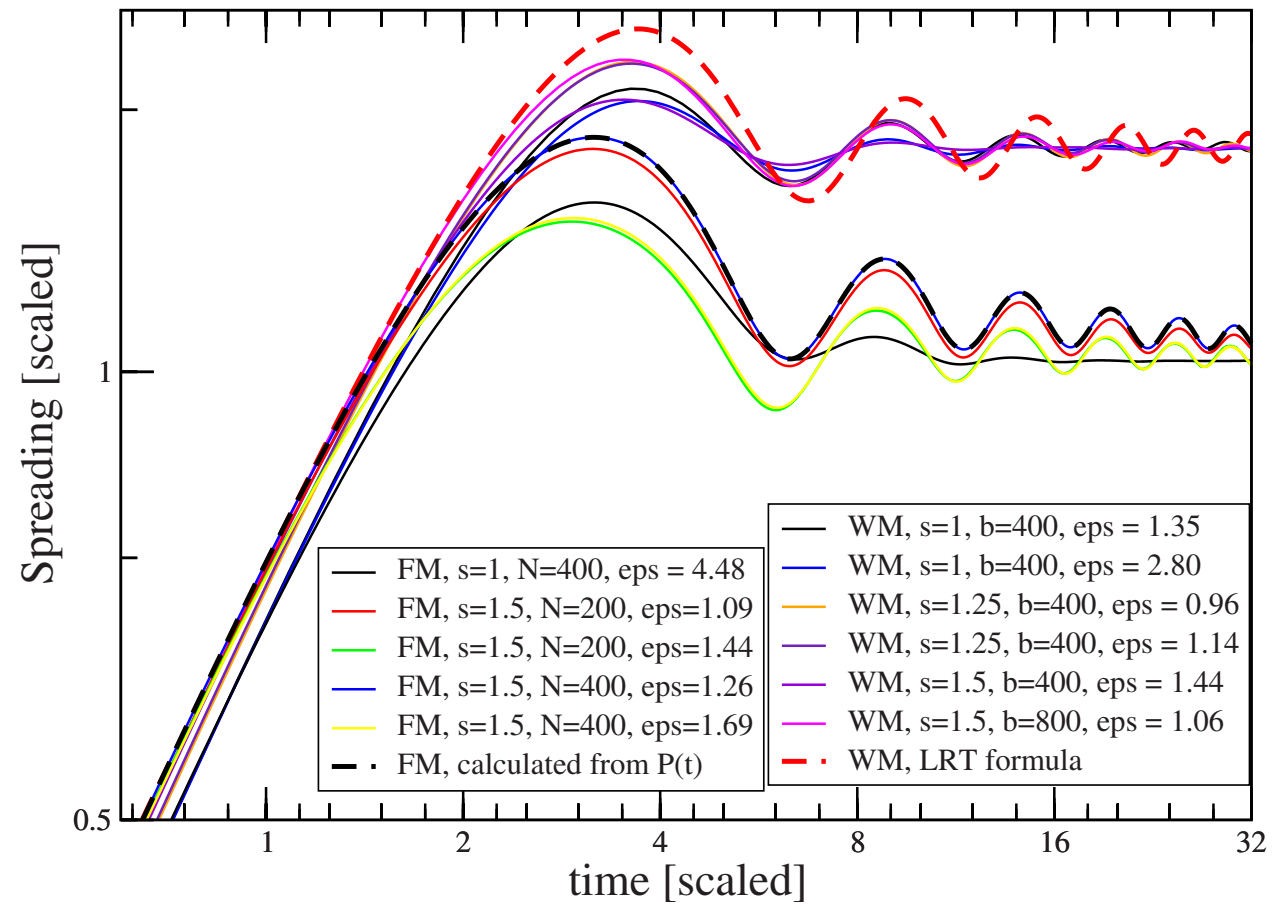

FIG. 10. (Color online) Scaled spread $\Delta E_{\mathrm{sprd}} /\left(\omega_{c}^{s} \epsilon^{2} / s\right)^{1 / 2}$ versus scaled time $\omega_{c} t$ for the FM and the WM. The theoretical predictions Eqs. (24) and (28) are plotted for comparison. 


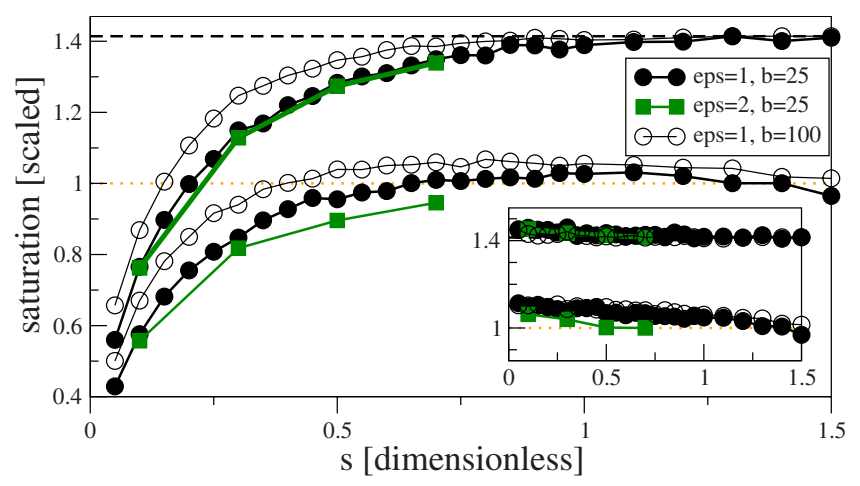

FIG. 11. (Color online) The scaled saturation value of $\Delta E_{\text {sprd }}$ for the FM (lower curves approach 1) and for the WM (upper curves approach $\sqrt{2}$ ). The saturation value falls down as $s$ becomes smaller due to the finite level spacing. To establish the latter statement we re-plot the same data but rescale the spread as $\Delta E_{\mathrm{sprd}} /\left[\left(\omega_{c}^{s} \epsilon^{2} / s\right)\right.$ $\left.-\left(\omega_{\varrho}^{s} \epsilon^{2} / s\right)\right]^{1 / 2}$.

$$
\begin{gathered}
c_{0}(t) \approx 1, \\
\dot{c}_{0}(t) \approx 0, \\
\ddot{c}_{0}(t) \approx-C(t),
\end{gathered}
$$

which demonstrates the agreement with Eq. (58). The numerical results in Fig. 10 confirm the validity of Eq. (68) for the FM and Eq. (58) for the WM. We note that in the FM case the effect of recurrences is more pronounced because they are better synchronized: all the out-in-out traffic goes exclusively through the initial state. Figure 11 establishes the $\sqrt{2}$ ratio throughout the whole range of $s$ values. One should be aware that for small $s$ the $\left(\omega_{c}^{s} / s\right)$ in Eq. (59) should be replaced by $\left(\omega_{c}^{s} / s\right)-\left(\omega_{\varrho}^{s} / s\right)$, which takes into account the finite level spacing in the numerical simulations. For very small $s$ this goes to $\log \left(\varrho \omega_{c}\right)$ as if $s=0$. The finite level spacing effect clearly shows up in the numerics and would not arise in the strict continuum limit.

\section{SUMMARY AND DISCUSSION}

In this paper, we have considered a quantum mechanical system, prepared in a discrete state that subsequently decays into a non-Ohmic continuum of other states. Two different models that have the same spectral properties but still different underlying dynamics have been analyzed and the respective results have been critically compared. One model (FM) reflects integrable dynamics while the other is an RMT model (WM) that corresponds to chaotic dynamics. In both cases, a universal generalized Wigner time that governs the relaxation process has emerged, while the nonuniversality is reflected in the decay law: we find that for "non-Ohmic chaos" (WM case) the survival probability becomes a stretched exponential beyond the Wigner time scale, which is both surprising and interesting. This is contrasted with the "integrable" power-law decay that takes over in the long time limit (FM case) and obviously is very different from the Ohmic exponential result. Only the standard case of Ohmic band profile is fully universal.
TABLE I. The various results for the LDOS and for the survival probability at a glance.

\begin{tabular}{lcc}
\hline \hline Model, $s$ & LDOS & $P_{0}(t)$ \\
\hline FM, WM $s=1$ & Lorentzian & Exponential decay \\
WM, $0<s<2$ & Semicircle core+tails & $\begin{array}{c}\text { Exponential-like decay } \\
\text { Exponential-like followed } \\
\text { by power-law }\end{array}$ \\
FM, $s=2$ & Singular core+tails & bog $(t)$ decay \\
FM, WM, $s>2$ & Delta core+tails & No decay after transient \\
$s \leq 0$ & Core +low weight tails & Infrared dominated \\
\hline \hline
\end{tabular}

We have also investigated the temporal behavior of the second moment of the spreading wave packet. We have found that in the FM case the generalized Wigner time is reflected in the spreading process and not only in the survival probability, contrary to the naive linear-response theory expectation.

Non-Ohmic coupling to the continuum emerges in various frameworks in physics. Quantized chaotic systems might exhibit non-Ohmic fluctuations due to semi-classically implied long time power-law correlations, and in any case the typical power spectrum is in general not like "white noise" (e.g., [14-16]). Other examples [17] appear in the context of a many-particle system, where the hierarchy of states and associated couplings, ranging from the single-particle levels to the exponentially dense spectrum of complicated manyparticle excitations, can lead to a very structural non-Ohmic band profile describing the residual interactions. These nonuniversal structures of the band profile of the perturbation lead to a highly nonlinear decay of the survival probability. Depending on the context, the survival probability could be also related to the study of dephasing or indirectly to the study of quantum fidelity and irreversibility: the generalized Wigner time is reinterpreted as the coherence time in the same sense as in Landau's Fermi-liquid theory.

It is worth mentioning that in a bosonic second quantized language the decay of the probability can be reinterpreted as the decay of the site occupation $\hat{n}$. If the interaction between the bosons is neglected this reduction is exact and merely requires an appropriate dictionary. In the latter context each level becomes a bosonic site which is formally like an harmonic oscillator, and hence the initially empty continuum is regarded as a zero-temperature bath. Consequently the decay problem is formally reinterpreted as a quantum dissipation problem with an Ohmic $(s=1)$ or non-Ohmic $(s \neq 1)$ bath. The generalized Wigner time scale is associated with the damped motion of the generalized coordinate $\hat{n}$.

Table I summarizes the various results that we have obtained for the survival probability. We conclude this section with a somewhat technical discussion of the crossovers between the various $s$ regimes. Note that the statements below are implied by inspection of Fig. 4. The strictly universal Ohmic result holds for $s=1$. The super-Ohmic universal regime is bounded from above by $s=2$, but for finite ultraviolate cutoff $\omega_{c}$ the effective border is a bit lower. This means that the marginally universal $s=2$ behavior prevails in a finite strip around $s=2$. For $s>2$, if the coupling to the continuum 
is small enough, the survival probability does not decay, except a short transient that can be described by FOPT. Similarly, the sub-Ohmic universal regime is bounded from below by $s=0$, but for finite infrared cutoff the effective border is a bit higher. Below this $s$-border the FOPT tails of the LDOS become subdominant [as implied by the divergence of the first term in Eq. (55), and the decay of the survival probability becomes infrared determined: This means that the effective cutoff is not $\omega_{c}$, but some different ill-defined (model-dependent) cutoff at much lower frequencies that might be determined by the level spacing statistics.

\section{ACKNOWLEDGMENTS}

This work was supported by the U.S.-Israel Binational Science Foundation (BSF). Some preliminary calculations have been done by Ori Ben-Dayan under the supervision of D.C.

\section{APPENDIX A: GREEN'S-FUNCTION FORMULATION}

For convenience we take the energy reference as $E_{0}=0$. The resolvent is defined as

$$
G^{+}(\omega)=\frac{1}{\omega-\mathcal{H}+i 0} .
$$

Substitution of $\mathcal{H}=\mathcal{H}_{0}+V$, expansion to infinite order, and exact geometric summation, can be carried out in the FM case, leading to the following standard result:

$$
\left\langle 0\left|G^{+}(\omega)\right| 0\right\rangle=\frac{1}{\omega-\Delta(\omega)+i(\Gamma(\omega) / 2)} .
$$

Using the identity $\operatorname{Im}\left[G^{+}\right]=-\pi \delta(\omega-\mathcal{H})$ one realizes that the LDOS is given by the expression

$$
\rho(\omega)=-\frac{1}{\pi} \operatorname{Im}\left[\left\langle 0\left|G^{+}(\omega)\right| 0\right\rangle\right]
$$

leading to Eq. (26). The evolution operator is given as the FT of the resolvent, namely, $U(t)=\mathrm{FT}\left[-2 \operatorname{Im}\left[G^{+}(\omega)\right]\right]$, hence the survival amplitude is

$$
c_{0}(t)=\langle 0|U(t)| 0\rangle=\mathrm{FT}[2 \pi \rho(\omega)]
$$

in agreement with the elementary derivation in the text.

\section{APPENDIX B: OPTIONAL DERIVATION OF THE SURVIVAL AMPLITUDE FORMULA}

In this appendix we give an optional direct derivation for the survival amplitude formula in the FM case without relaying on the theory of Green's functions. We are interested in finding $c_{n}(t)$ and $c_{0}(t)$, which are the amplitudes to find the particle in the respective levels. The Schrodinger equation is

$$
i \frac{\partial c_{0}}{\partial t}=\sum_{n} e^{-i \omega_{n} t} V_{0, n} c_{n}(t),
$$

$$
i \frac{\partial c_{n}}{\partial t}=e^{i \omega_{n} t} V_{n, 0} c_{0}(t),
$$

where $\omega_{n} \equiv E_{n}-E_{0}$. By integration over Eq. (B2) we get

$$
c_{n}(t)=-\int_{0}^{t} e^{i \omega_{n} t^{\prime}} V_{n, 0} c_{0}\left(t^{\prime}\right) d t^{\prime}
$$

placing Eq. (B3) into Eq. (B1) we get

$$
\frac{d c_{0}}{d t}=-\int_{0}^{t} C\left(t-t^{\prime}\right) c_{0}\left(t^{\prime}\right) d t^{\prime},
$$

where

$$
C(\tau)=\operatorname{FT}[\tilde{C}(\omega)]=\sum_{n}\left|V_{n, 0}\right|^{2} e^{-i \omega_{n} \tau}
$$

We want to solve the survival amplitude equation using a Laplace transform technique. For that purpose we define $K(\tau)=\Theta(\tau) C(\tau)$, where $\Theta(\tau)$ is the Heaviside step function. Then we rewrite the equations as

$$
\frac{d c_{0}}{d t}=\delta(t)-\int_{-\infty}^{+\infty} K\left(t-t^{\prime}\right) c_{0}\left(t^{\prime}\right) d t^{\prime},
$$

where $c_{0}(\tau)$ is zero for negative $\tau$ and unknown for positive $\tau$. The corresponding equation for the Fourier components is

$$
-i \omega c_{\omega}=1-\tilde{K}(\omega) c_{\omega} .
$$

From here it follows that the survival amplitude can be written as an FT

$$
c_{0}(t)=\int_{-\infty}^{+\infty} \frac{d \omega}{2 \pi}\left[\frac{e^{-i \omega t}}{-i \omega+\tilde{K}(\omega)}\right] .
$$

From the definition of $K(\tau)$ and using the convolution theorem it follows that

$$
\tilde{K}(\omega)=\frac{1}{2} \Gamma(\omega)+i \Delta(\omega) .
$$

Hence consistency with Eq. (17) is established.

\section{APPENDIX C: THE ENERGY SPREADING FORMULA}

For the derivation of the energy spreading formula Eq. (56) it is convenient to regard the Hamiltonian as time dependent,

$$
\mathcal{H}[\lambda(t)]=\mathcal{H}_{0}+\lambda(t) V,
$$

where $\lambda(t)$ is a time-dependent parameter. We define generalized forces in the standard way

$$
\mathcal{F}=-\frac{\partial \mathcal{H}}{\partial \lambda}(\lambda)
$$

Using the Heisenberg picture and the usual notation $\mathcal{F}(t)$ $=U(t)^{-1} \mathcal{F} U(t)$, we have the following relation: 


$$
\frac{\partial \mathcal{H}}{\partial t}=-\dot{\lambda}(t) \mathcal{F}(t)
$$

Thus the change in the energy can be written as

$$
\mathcal{H}(t)-\mathcal{H}(0)=-\int_{0}^{t} \dot{\lambda}\left(t^{\prime}\right) \mathcal{F}\left(t^{\prime}\right) d t^{\prime}
$$

Squaring and taking the expectation value with respect to the initial state we get

$$
\left[\Delta E_{\mathrm{sprd}}(t)\right]^{2}=\int_{0}^{t} d t^{\prime} \int_{0}^{t} d t^{\prime \prime} \dot{\lambda}\left(t^{\prime}\right) \dot{\lambda}\left(t^{\prime \prime}\right) C\left(t^{\prime}, t^{\prime \prime}\right),
$$

where $C\left(t^{\prime}, t^{\prime \prime}\right)=\left\langle\mathcal{F}\left(t^{\prime}\right) \mathcal{F}\left(t^{\prime \prime}\right)\right\rangle$ is the autocorrelation function. The free evolution during the time interval $[0, t]$ corresponds formally to a "rectangular pulse"

$$
\lambda\left(t^{\prime}\right)=\Theta\left(t^{\prime}\right)-\Theta\left(t^{\prime}-t\right),
$$

where $\Theta()$ is a Heaviside step function. Its time derivative is $\dot{\lambda}=\delta\left(t^{\prime}\right)-\delta\left(t^{\prime}-t\right)$, leading to Eq. (56).
[1] N. Auerbach and V. Zelevinsky, Phys. Rev. C 65, 034601 (2002); V. V. Sokolov and V. G. Zelevinsky, Nucl. Phys. A 504, 562 (1989).

[2] C. Cohen-Tannoudji, J. Dupont-Roc, and G. Grynberg, AtomsPhoton Interactions: Basic Processes and Applications (Wiley, New York, 1992).

[3] M. A. Nielsen and I. L. Chuang, Quantum Computation and Quantum Information (Cambridge University Press, Cambridge, 2000).

[4] V. N. Prigodin, B. L. Altshuler, K. B. Efetov, and S. Iida, Phys. Rev. Lett. 72, 546 (1994); B. L. Altshuler, Y. Gefen, A. Kamenev, and L. S. Levitov, ibid. 78, 2803 (1997).

[5] C. W. J. Beenakker and H. van Houton, in Solid State Physics: Advances in Research and Applications, edited by H. Ehrenreich and D. Turnbull (Academic, New York, 1991), Vol. 44, pp. $1-228$.

[6] E. Persson, I. Rotter, H.-J. Stöckmann, and M. Barth, Phys. Rev. Lett. 85, 2478 (2000).

[7] V. Weisskopf and E. P. Wigner, Z. Phys. 63, 54 (1930).

[8] E. Wigner, Ann. Math 62, 548 (1955); 65, 203 (1957).

[9] M. Feingold, Europhys. Lett. 17, 97 (1992).

[10] V. V. Flambaum, A. A. Gribakina, G. F. Gribakin, and M. G. Kozlov, Phys. Rev. A 50, 267 (1994).
[11] F. M. Izrailev and A. Castaneda-Mendoza, Phys. Lett. A 350, 355 (2006); V. V. Flambaum and F. M. Izrailev, Phys. Rev. E 64, 026124 (2001); 61, 2539 (2000).

[12] Y. V. Fyodorov, O. A. Chubykalo, F. M. Izrailev, and G. Casati, Phys. Rev. Lett. 76, 1603 (1996).

[13] J. L. Gruver, J. Aliaga, H. A. Cerdeira, P. A. Mello, and A. N. Proto, Phys. Rev. E 55, 6370 (1997).

[14] D. Cohen and E. J. Heller, Phys. Rev. Lett. 84, 2841 (2000).

[15] D. Cohen and T. Kottos, Phys. Rev. E 63, 036203 (2001).

[16] M. Hiller, D. Cohen, T. Geisel, and T. Kottos, Ann. Phys. 321, 1025 (2006).

[17] P. G. Silvestrov, Phys. Rev. B 64, 113309 (2001); A. Amir, Y. Oreg, Y. Imry, Phys. Rev. A 77, 050101(R) (2008).

[18] F. Haake, Quantum Signatures of Chaos (Springer, New York, 2000).

[19] K. O. Friedrichs, Commun. Pure Appl. Math. 1, 361 (1948).

[20] D. Cohen, F. M. Izrailev, and T. Kottos, Phys. Rev. Lett. 84, 2052 (2000).

[21] D. Cohen, Ann. Phys. 283, 175 (2000).

[22] M. Hiller, T. Kottos, and T. Geisel, Phys. Rev. A 73, 061604 (2006).

[23] T. Kottos and D. Cohen, Europhys. Lett. 61, 431 (2003). 